\title{
Agnizing Sarcopenia and Coherent Variable Optimization of Body Fat Percentage using Genetic Algorithms and Regression
}

\author{
J. Grace Hannah, D. Gladis
}

\begin{abstract}
Obesity is a malady which poses wide threats across the world with its augmented inflation. A domineering determinant to most pandemic diseases in the human body is the agglomeration of body fat. Therefore, an apposite anatomization of body fat estimation for every individual is incumbent. The previous work aberrates and pioneered the implementation of attributes from the lipid profile and Bio-Electric Impedance Analysis (BIA) method of a person, from the conventional use of attributes such as BMI, age and gender to obtain the value of body fat percentage. But the proposed analysis meliorates the accuracy of body fat percentage and resuscitated the gamut of health gremlins it vanguards to. This paper also delineates the variable optimization using regression and genetic algorithm for the attributes incorporated to procure the body fat percentage. Thereby corroborating and revamping the veracity of the novel body fat percentage derived using lipids and the BIA method. The study has further helped in diagnosing a disease known as sarcopenia. The samples from the blood tests and Bio-Electric Impedance method have been procured from the Institute of Bio-Chemistry, after obtaining the consent from the Institutional Ethics Committee, Madras Medical College, Chennai. The simulations are carried out in MATLAB GUI and the results have been successfully obtained.
\end{abstract}

Keywords: Obesity, Body Fat Percentage, Bio-Electric Impedance, Sarcopenia, MATLAB GUI

\section{INTRODUCTION}

A euphemism extensively used and addressed with paramount concern around the globe for body fat is Obesity. The World Health Organization (WHO) declared that a whooping number of about 1.9 billion adults were overweight, 650 million were considered to be obese ${ }^{\text {[19] }}$, [25], [26] and about $80 \%$ of them have type 2 diabetes and other consanguineous ailments. Bariatric is the branch of medicine that deals with the surgical treatments of obesity ${ }^{[3]}$, [20] and its cognate disorders. The bariatric medicaments utilized in the recent years for mitigating these disorders have evolved multifariously. However, the phase of life where an individual tends to put on the avoirdupois is that measure which would determine their potential to

Revised Manuscript Received on December 30, 2019.

* Correspondence Author

J. Grace Hannah*, Ph.D Research Scholar, Computer Science, University of Madras.

Dr. D. Gladis, Principal, Bharathi Women's College, Chennai.

(C) The Authors. Published by Blue Eyes Intelligence Engineering and Sciences Publication (BEIESP). This is an open access article under the CC BY-NC-ND license (http://creativecommons.org/licenses/by-nc-nd/4.0/) metamorphose back to normal. The calorie disparity in the ingestion, and the energy expended by an individual has engendered the amassing of detrimental adipose tissues in the human body.

The rate at which obesity affects human beings is colossal to that of infirmities caused due to underweight. An individual having a higher waist-hip ratio and weighing more than the reckoned standard of measure usually finds it arduous to lose weight. The cognizance of obesity is concomitant to adipose tissues, and these tissues are multifaceted and contemplated as a complex organ [21], [23]. These adipose tissues bifurcate into White Adipose Tissues (WAT) and Brown Adipose Tissues (BAT) [1], [2], [25], [26]. Besides operating as a system for caching the nimiety of energy, defense against nippy, frigid conditions and the perils confronting our day to day lives, adipose tissues are responsible to generate a conglomeration of molecular emissaries called as adipokines [6], [7]. These adipokines steers an eclectic medley of functions comprising of appetite, fertility, neural development, inflammatory responses and other hormonal operations such as insulin [22], [23]. In spite of the series of sanguine functions, the uncurbed energy and the amassment of white adipose tissues steers the cause to menacing physical impairments and disparate health encumbrances. Although multifarious studies depict that the fat cells are waned only in proportion, obliterating the fat cells which are accumulated becomes an increasingly onerous task ${ }^{[20],[4]}$. Due to these predicaments, the analysis of fat percentage in the human body is rudimentary to evince the health fettle of an individual ${ }^{[8],[9]}$.

This paper aims to ascertain the need for an empirical examination of body fat related to an individual's lipid profile and the subdivided fat values by using regression and genetic algorithms. Thus, the optimization of parameters in the body fat formula escalates the accuracy of body fat percentage, thereby enabling to fathom the occurrence of sarcopenia in individuals having higher body fat percentage. The paper is structured into sections, where Section II gives a brief description of the techniques executed and the factors obtained from it. Section III elucidates the optimized formula for body fat percentage along with the techniques used. Section IV demonstrates the results obtained in MATLAB, and Section V delineates the compendium and conclusion of the work done. 


\section{METHODOLOGY}

The samples of blood tests and factors from the BIA method were drawn from adults aged between 20- 75 years. The population of study were circumscribed to obese, hyperlipidemic patients who were confronted with health gremlins ascribed to agglomeration of blubber in the body.

This study enumerates the formulation of body fat percentage by computation of the values procured from the blood tests and the Bioelectric Impedance Analysis (BIA) method for an individual.
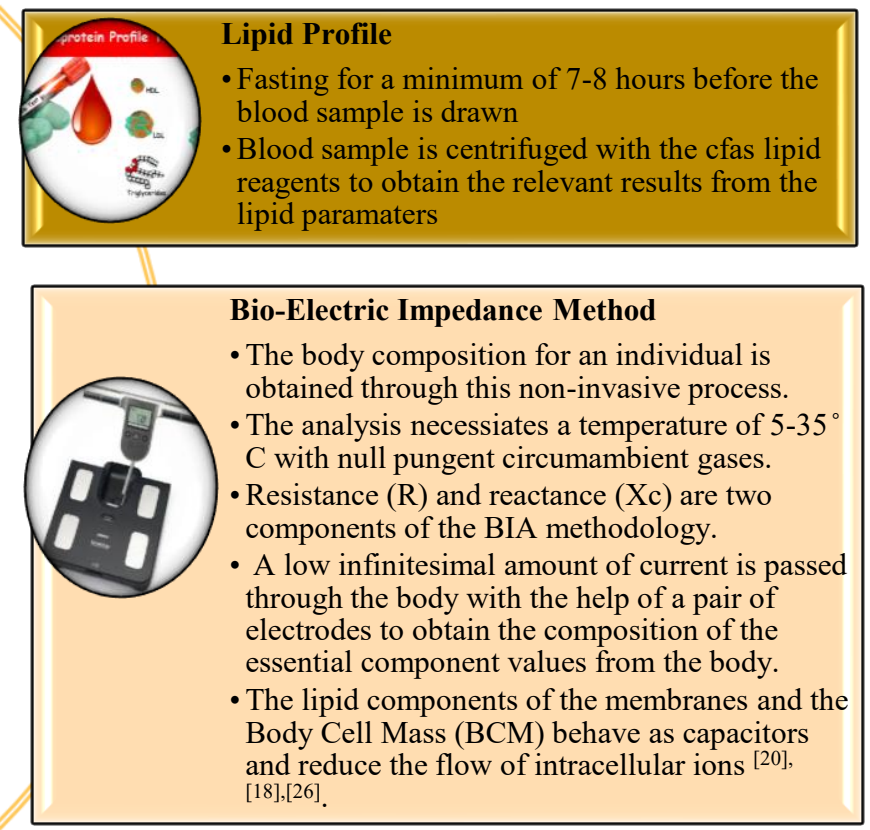

Fig 1: Methods used to procure data samples

The above two methodologies are ostracized for individuals who were anaemic and suffering from other pernicious health disorders, parturient women and patients with hemorrhage dysfunctions. The lipid profile tests and the BIA method catalogues a list of components for each individual which helps in analyzing the state of health of an individual [16], [26]. Both the above tests also aids to sift the distribution of fat cells in the body and delineates the following factors: Total Cholesterol (TC), High-density Lipoprotein (HDL), Low density Lipoprotein (LDL) and Triglycerides (TG) are fragmented integrants of the former, whereas the latter test generates values for the modules such as subcutaneous fat, visceral fat percentage, the concomitant age corresponding to the fat percentage, Body Mass Index (BMI) and the muscle mass percentage for every individual ${ }^{[20],[26]}$.

\section{OPTIMIZATION OF THE FORMULA AND TECHNIQUES USED}

The existing body fat formula is given as [14],[20],[25],[26]:

$$
[(1.2 * B M I)+(0.23 * \text { Age })-(10.8 * \text { Gender })-5.4]
$$

The conventional formula derived by Paul Duerenberg has been permeated to anatomize the fat percentage in the human body. But it has been ascertained that for a person of herculean build with exceeding muscle mass, there arouses an unwarranted surge in the BMI, ensuing that the individual falls in the class of anamously obese or overweight compartment. Therefore, to circumvent errors and to proffer an unswerving precise result, a novel formula comprising of Total Cholesterol (TC) from the lipid profile tests, subcutaneous and visceral fat from the BIA method, age pertaining to the fat accumulation in the body and gender of the person are taken into consideration [20],[26]

$$
\begin{array}{r}
\text { Body fat } \%=[0.5(F D)+(0.03(B I A A-A))+ \\
(0.02 * C H)-(10.9 * G E N)+10.8]
\end{array}
$$

Where FD denotes the sum of the total fat deposit from the subcutaneous and the visceral fat in the human body. The difference between the age pertaining to fat in the body and the original age is then computed. The total cholesterol obtained from the lipid profile is denoted as $\mathrm{CH}$, and gender (GEN) takes the value of 0 for female, and 1 for male [20], [26].

\section{A) REGRESSION \& GENETIC ALGORITHM}

Variable optimization is an integral part of meliorating the coherent body fat formula. Therefore, in order to correlate the derived formula and to obtain impeccable results, the curve fitting model along with genetic algorithm has been implemented. Parameter selection is implemented by performing a constraint-based optimization where a lower boundary and an upper boundary are set. Genetic operators through a fitness function is first used for the selection of strong chromosomes, the next step involves the entwining of chromosomes by using the arithmetic cross over method with the cross over probability set to 0.8 . The final step of genetic operation uses mutation to randomly commute the genes from the selected pair of chromosomes. The mutation probability is set to 0.2 and the number of generations to be iterated is set to 1000 with the elite count set to 4 . Optimization occurs with the number of generations and the best fitness value and the mean fitness value are obtained.

Least - Square regression method is applied to fit the curve between the observed and the predicted values. Initially the predicted values are found by inversing the original data matrix and by applying the multiplication of the matrix with the unknown parameters. Then the mean square error is found by squaring the difference between the original data and the predicted values.

\section{B) IDENTIFICATION OF SARCOPENIA USING}

\section{THE DERIVED FORMULA}

Sarcopenia is a term which refers to the loss of muscle mass [24]. The meliorated formula thus obtained after genetic operation is applied to acquire the precise body fat percentage for each person. The next step involves obtaining the muscle mass percentage from the BIA method for every individual.

It has been studied that when the formula has been contoured using genetic algorithm, the values for muscle mass percentage amortizes with the escalation of body fat percentage. 
Table- I: Analysis of Sarcopenia using the Existing Body Fat Formula

\begin{tabular}{|c|c|c|c|c|}
\hline Gen & Age & $\begin{array}{c}\text { Existing } \\
\text { Formula }\end{array}$ & Muscle Mass & $\begin{array}{c}\text { Sarcopenia } \\
\text { Analysis }\end{array}$ \\
\hline 1 & 25 & 24.58 & 27.60 & Normal \\
\hline 0 & 49 & 43.33 & 21.20 & Positive \\
\hline 1 & 54 & 33.54 & 25.20 & Normal \\
\hline 1 & 60 & 29.16 & 24.32 & Normal \\
\hline 0 & 52 & 40.52 & 23.32 & Positive \\
\hline 0 & 54 & 24.58 & 35.72 & Normal \\
\hline
\end{tabular}

Table- II: Analysis of Sarcopenia using the derived Body Fat Formula

\begin{tabular}{|c|c|c|c|c|}
\hline Gen & Age & $\begin{array}{c}\text { Derived } \\
\text { Formula }\end{array}$ & Muscle Mass & $\begin{array}{c}\text { Sarcopenia } \\
\text { Analysis }\end{array}$ \\
\hline 1 & 25 & 30.41 & 20.20 & Positive \\
\hline 0 & 49 & 54.09 & 16.60 & Positive \\
\hline 1 & 54 & 40.98 & 21.40 & Positive \\
\hline 1 & 60 & 24.73 & 26.50 & Normal \\
\hline 0 & 52 & 46.40 & 19.40 & Positive \\
\hline 0 & 54 & 44.51 & 22.70 & Positive \\
\hline
\end{tabular}

Tables I and II shows the muscle mass percentage with relation to the body fat percentage of an individual garnered from the Bio-Electric Impedance Analysis. The tables patently expound that with the existing formula, the recognition and extant of sarcopenia is misclassified due to the absence of tangible attributes and variable optimization.

\section{RESULTS OBTAINED}

The result executed in the subsequent MATLAB GUI figure shows the optimization of the attributes is implemented through the genetic operators over a period of generations (iterations). The mean error rate is obtained after regression to assay the accuracy of the derived novel formula.

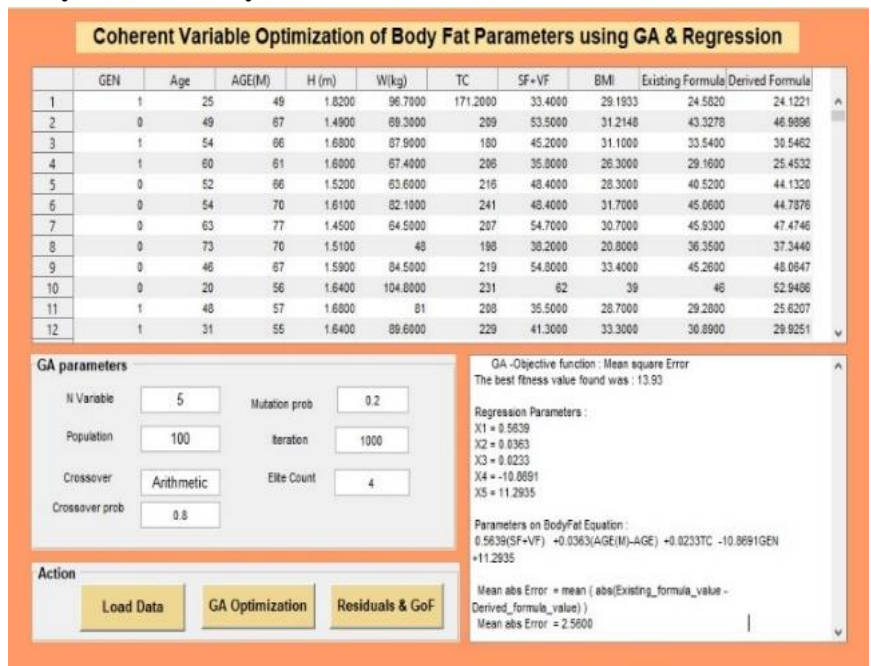

Fig 1. Variable Optimization using Genetic Algorithm and mean error using Regression.

\section{CONCLUSION}

The above indagation has aided in the cognizance of interdependency between body fat, lipids and muscle mass percentage of an individual. It has helped to ameliorate the accuracy of the body fat percentage by parameter optimization using genetic algorithm and regression. A mean absolute error rate of $0.5 \%$ has proved that the novel formula derived after variable optimization can be equated to be used as a standard measure. This study also evinced that when the lipids in the blood depletes, the fat mass burgeons copiously thereby indirectly affecting and attenuating the muscle mass percentage of a person. Thus, the implemented indagation has piloted the emphasis on variable selection and optimization as a crucial factor in order to perceive health snags expeditiously. An ensuing elaborate analysis on sarcopenia is being contemplated.

\section{REFERENCES}

1. R Drolet, C Richard, et al, "Hypertrophy and hyperplasia of abdominal adipose tissues in women", International Journal of Obesity (2008) 32, 283-291; doi: 10.1038/sj.ijo.0803708; published online 28 August 2007.

2. [2] Carolina T. Mendes- dos-Santos, et al, "Normalization of height and excess body fat in children with salt-wasting 21-hydroxylase deficiency", J. Pediatr, (Rio J.) vol.87 no.3 Porto Alegre May/June 2011

3. https://www.niddk.nih.gov/health information/weight-management/bariatric-surgery/definition-facts, Definition \& Facts for Bariatric Surgery.

4. Lester b. Salans, et al, "Studies of Human Adipose Tissue, Adipose cell size and Number in non obese and obese patients", The Jour. of Clinical Investigation, Vol 52.

5. http://www.who.int/mediacentre/factsheets/fs311/en/obesity and overweight

6. David A. Bernlohr, et al, “Adipose tissue and lipid metabolism”, Elsevier Science, 2002.

7. Christoph H. Saely, Kathrin Geiger, Heinz Drexel, "Brown versus White Adipose Tissue: A Mini-Review”, Gerontology 2012

8. ClÁudia M. Oller Do Nascimento, et al, "Metabolism and secretory function of white adipose tissue: Effect of dietary fat", Anais da Academia Brasileira de Ciências (2009) 81(3): 453-466.

9. Health risks of being Overweight, U.S. Department of Health and Human Services, October 2007.

10. Rômulo A. Fernandes, et al, "The use of bioelectrical impedance to detect excess visceral and subcutaneous fat", Journal de Pediatria, August, 2007.

11. Fredrik Toss, "Body fat distribution, inflammation and cardiovascular disease", ISSN: 0346-6612; Sweden, 2011

12. Chun-Hao Chen, et al, "The study of Anthropometric estimates in the Visceral fat of healthy individuals", Nutrition Journal, 2014.

13. Leigh Peele, "Body Fat Percentage: A Complete Guide to Evaluation and Measurement", 2010

14. Paul Deurenberg, et al, "Singaporean Chinese adolescents have more subcutaneous adipose tissue than Dutch Caucasians of the same age and body mass index", Asia Pacific J Clin Nutr 2003;12 (3):261-265

15. Sun SS, Chumlea WC, Heymsfield SB, "Development of Bioelectric impedance analysis prediction equations for body composition with the use of multicomponent model for use in epidemlogic surveys", American Society for clinical Nutrition.

16. DK Key, Bosaeus, "Body Composition estimated by Bioelectric impedance in the Swedish elderly

Development of population - based prediction equation and reference values of fat - free mass and body fat for 70 - 75 year olds ", European Journal of Clinical Nutrition.

17. Linda L.D. Zhong, Wai Kun et al, "The combination effects of body acupuncture and auricular acupressure compared to sham acupuncture for body weight control: Study protocol for a randomized controlled trial", BioMed Central.

18. Mahshjid Dehgan et al, "Is Bioelectric Impedance accurate for use in large epidemilogical studies?” BioMed Central, Nutritional Journal, 2008

19. https://www.who.int/factsonobesity

20. J. Grace Hannah, D.Gladis, "A Statistical Indagation of Body Fat Percentage: A Sift Cognitive Correlation of Lipid Data and Bio-Electric Impedance Analysis in Humans", International Journal of Advances in Science Engineering \& Technology, April 2018.

21. S. Cinti, "The adipose organ at a glance, Dis. Model Mech", 5(2012) 588-594

22. N.Ouchi, J.L. Parker, J.J Lugus, K.Walsh, Nat. "Adipokines in Inflammation and metabolic disease”, Rev. Immunol, 11(2011) 85-97.

Published By:

Blue Eyes Intelligence Engineering \& Sciences Publication

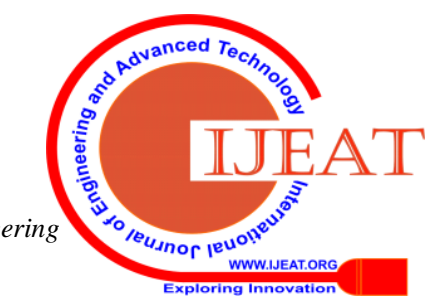


23. E.L.Thomas, et al, "Whole Body Fat: Content and Distribution", Progress in Nuclear Magnetic Resonance Spectroscopy, 73 (2013), 56-80.

24. Rita Rastogi Kalyani et al, "Age-Related and Disease related Muscle mass: The effect of diabetes, Obesity and other diseases", The lancet Diabetes \& Endocrinology, Vol 2, Issue 10, P819-829, Oct 2014

25. J. Grace Hannah, D. Gladis, "Aggrandizing the Accuracy of Body Fat Percentage by Stratification using Decision Tree", International Journal of Innovative Technology and Exploring Engineering (IJITEE), ISSN: 2278-3075, Volume-8, Issue-11, September 2019, PP: 1883-1886

26. J. Grace Hannah, D. Gladis, "An Entropy based Classification of Body Fat using Fuzzy Rules commingled with Genetic Algorithm", International Journal of Recent Technology and Engineering (IJRTE), ISSN: 2277-3878, Vol-8, Issue-3, September 2019, PP: 2493-2496

\section{AUTHORS PROFILE}

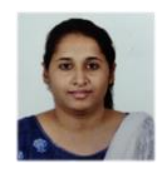

J. Grace hannah is a research scholar pursuing her Ph.D in Computer Science from the University of Madras. She has more than eight research papers to her credit, and has participated in various National and International conferences.

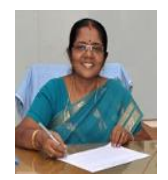

Dr. D. Gladis is the Principal of Bharathi Women's College, Chennai. She has headed the Department of Computer Science \& Research Institute in Presidency College, Chennai. She has guided more than fifteen research scholars under the M.Phil and Ph.D courses and has been an active researcher. Her area of interests are Neural Networks, Image Processing, Data Mining to name a few 